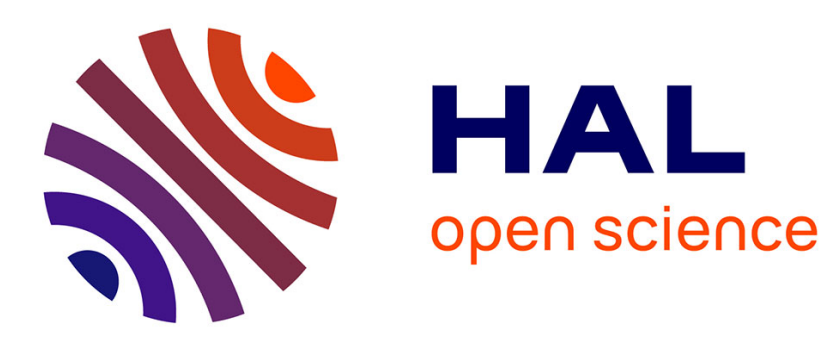

\title{
A new non-intrusive assessment method for VoLTE quality based on extended E-model
}

\author{
Duy Huy Nguyen, Hang Nguyen, Eric Renault
}

\section{To cite this version:}

Duy Huy Nguyen, Hang Nguyen, Eric Renault. A new non-intrusive assessment method for VoLTE quality based on extended E-model. MSPN 2016: 2nd international conference on Mobile, Secure and Programmable Networking, Jun 2016, Paris, France. pp.122 - 136, 10.1007/978-3-319-50463-6_11. hal-01446945

\section{HAL Id: hal-01446945 \\ https://hal.science/hal-01446945}

Submitted on 26 Jan 2017

HAL is a multi-disciplinary open access archive for the deposit and dissemination of scientific research documents, whether they are published or not. The documents may come from teaching and research institutions in France or abroad, or from public or private research centers.
L'archive ouverte pluridisciplinaire HAL, est destinée au dépôt et à la diffusion de documents scientifiques de niveau recherche, publiés ou non, émanant des établissements d'enseignement et de recherche français ou étrangers, des laboratoires publics ou privés. 


\title{
A new non-intrusive assessment method for VoLTE quality based on extended E-model
}

\author{
Duy-Huy Nguyen, Hang Nguyen, and Éric Renault \\ SAMOVAR, Télécom SudParis, CNRS, Université Paris-Saclay, \\ 9 rue Charles Fourier - 91011 Evry Cedex \\ \{duy_huy.nguyen, hang.nguyen, eric.renault\}@telecom-sudparis.eu
}

\begin{abstract}
Voice over Long Term Evolution (called VoLTE) is always a main service that brings a big benefit for mobile operators. However, the deployment of VoLTE is very complex, specially for guaranteeing of Quality of Service (QoS) to meet quality of experience of mobile users. The key purpose of this paper is to present an object, non-intrusive prediction model for VoLTE quality based on LTE-Sim framework and the extended E-model. This combination allows to overcome the lack of the E-model is that how to determine exactly its inputs. Besides, we propose to add the jitter buffer as an essential input factor to the E-model. The simulation results show that our proposed model can predict voice quality in LTE network via both the E-model and the Extended E-model. The proposed model does not refer to the original signal, thus, it is very suitable for predicting voice quality in LTE network for many different scenarios which are configured in the LTE-Sim framework. The simulation results also show that the effect of jitter buffer on user perception is very significant.
\end{abstract}

Keywords: VoLTE, voice quality, LTE, Extended E-model, MOS.

\section{Introduction}

LTE network is developed by the Third Generation Partnership Project (3GPP) [1]. It is a mobile network which has high data rates, low latency and fully packet-based. It means to improve the capability of legacy system by increasing data rates and extending superior QoS for various multimedia applications. Voice over LTE network (called VoLTE) is a main service of LTE network. Since LTE network is a full packet-switched, thus, the deployment of VoLTE service is very complicated. All voice traffics over LTE network are VoIP (Voice over Internet Protocol) including VoLTE. According to [2], there are two types of voice traffic over LTE network, those are VoLTE and VoIP. VoLTE is really the VoIP service with QoS guaranteed [2]. Table 1 represents the service classes in LTE network where voice service is a Guaranteed Bit Rate (GRB) service which has the second priority just after IP Multimedia Subsystem (IMS) signaling. However, in order to guarantee VoLTE quality is an extreme challenge. 
Table 1. LTE service classes with QoS requirements

\begin{tabular}{|c|c|c|c|c|}
\hline $\begin{array}{l}\text { Resource } \\
\text { Type }\end{array}$ & Priority & \begin{tabular}{|c|} 
Packet \\
Delay \\
Budget \\
$(\mathbf{m s})$
\end{tabular} & \begin{tabular}{|c|} 
Packet \\
Error \\
Loss \\
Rate \\
\end{tabular} & Example services \\
\hline \multirow{4}{*}{$\begin{array}{c}\text { Guaranteed } \\
\text { Bit Rate } \\
\text { (GBR) }\end{array}$} & 2 & 100 & $10^{-2}$ & Conversational voice \\
\hline & 4 & 150 & $10^{-3}$ & $\begin{array}{l}\text { Conversational video } \\
\text { (live streaming) }\end{array}$ \\
\hline & 3 & 50 & $10^{-3}$ & Real-time gaming \\
\hline & 5 & 300 & $10^{-6}$ & $\begin{array}{l}\text { Non-conversational video } \\
\text { (buffered stream) }\end{array}$ \\
\hline \multirow{5}{*}{ Non-GBR } & 1 & 100 & $10^{-3}$ & IMS signaling \\
\hline & 6 & 300 & $10^{-6}$ & $\begin{array}{l}\text { Video (buffered streaming) TCP-based } \\
\text { (e.g. www, e-mail, chat, FTP, P2P sharing, progressive video, etc.) }\end{array}$ \\
\hline & 7 & 100 & $10^{-6}$ & Voice, Video (live streaming, Interactive Gaming) \\
\hline & 8 & \multirow{2}{*}{300} & $10^{-3}$ & Video (buffered streaming) TCP-based \\
\hline & 9 & & $10^{-6}$ & (e.g. www, e-mail, chat, FTP, P2P sharing, progressive video, etc.) \\
\hline
\end{tabular}

In communications systems, the perceived voice quality is usually represented as the MOS. MOS can be attained by many methods. These methods are divided into two groups called subjective methods and objective ones. Subjective methods humans listening to a live stream or a recorded file and rating it on a ratio of 1 (poor) to 5 (excellent) [3]. These methods have some disadvantages such as too expensive, time consuming and are not suitable for a large network infrastructure. Otherwise, objective methods have more advantages, they eliminate the limitations of subjective methods. Objective methods are classified into two approaches: intrusive and non-intrusive ones. The intrusive methods (e.g. Perceptual evaluation of speech quality (PESQ) [4]) are more exact and are widely utilized to predict aware voice quality. However, they are not suitable for real-time services such as VoIP because they require original signals to refer. The non-intrusive methods (e.g. ITU-T E-model [5]) are computational models that are used for transmission planning purposes. They are not as accurate as the intrusive approaches and they do not have complex mathematical operations. The obtained results from objective methods do not always well relate to human perception. The main advantage of the non-intrusive methods are they predict voice quality without any reference to the original signals and they require less parameters than the intrusive methods. For several typical non-intrusive methods, authors in [6] proposed to use Random Neural Network (RNN) to assess voice quality over internet. Voice quality assessment was predicted in [7] using RNN. Another non-intrusive method was proposed in [8] based on RNN for evaluating video quality in LTE network. Authors in [9] proposed to use Wideband (WB) E-model to predict VoLTE quality for minimizing redundant bits generated by channel coding. In [10], authors investigated the effects of PLR and delay jitter on VoIP quality to assess prediction errors of MOS for the E-model. A new model called "Packet-E-Model" was proposed in [11] to measure speech quality perception for VoIP in Wimax network. In [12], authors presented a voice quality measurement tool based on the E-model. A framework of objective assessment method for estimating conversational quality in VoIP was proposed in [13]. In [14], a simplified versions of the E-model were proposed to simplify the 
calculations and focus on the most important factors required for monitoring the call quality. According to our knowledge, at present, there are not any proposals which allow to predict VoLTE quality using LTE-Sim framework and extended E-model.

We see that, the lack of the E-model is how to measure exactly the its input parameters. Therefore, we need a method to measure the input factors of the Emodel. In this paper, we propose to use the LTE-Sim [15] to calculate delay and PLR for voice users. LTE-Sim is a famous framework which allows to simulate entire LTE network, thus, it is quite similar to a real system. Outputs of LTESim are Delay and PLR which are essential input parameters of the E-model. In addition, we also take effects of network jitter into account by considering jitter parameter $\left(I_{j}\right)$ as an input parameter of the E-model. In order to obtain more real results, we simulate voice service with mobility in LTE heterogeneous network.

The rest of this paper is organized as follows: Overview of the system model is described in section 2 . In section 3 , we present the proposed model. The simulation results and performance evaluation of the proposed model are analysed in section 4 . The conclusion and future work is represented in section 5 .

\section{The system model}

\subsection{The LTE-Sim framework}

LTE-Sim is an open-source framework which is developed by Giuseppe Piro and his colleagues [15]. It is freely available for scientific community. It is used to simulate entire LTE network. There are many researchers who used LTESim to simulate their proposals such as scheduling strategies, radio resource optimization, frequency reuse techniques, the adaptive modulation and coding module, user mobility, and etc. for both downlink and uplink directions and in both multicell/multiuser environments. The implemented protocol stack of LTESim is represented on Figure 1 for both user-plane and control-plane, thus, it is nearly similar to the real LTE system.

Figure 1 can be briefly described as follows: When a voice traffic flow transmitted over the LTE-Sim, it is encapsulated sequentially with network protocols. For the downlink direction, the VoLTE packet uses transport protocols of Realtime Transport Protocol (RTP), User Datagram Protocol (UDP) and Internet Protocol (IP). It is then packetized with radio protocols such as Packet Data Convergence Protocol (PDCP), Radio Link Control (RLC) and Medium Access Control (MAC), and Physical (PHY) layer before it is transmitted over the air interface. LTE-Sim supports both IPv4 and IPv6 protocols with header sizes are 40 and 60 , respectively while the voice payload is about 32 bytes, thus, to reduce the overhead, Robust Header Compression ( $\mathrm{RoHC}$ ) is deployed at PDCP layer. The IP header is then compressed by RoHC down to only 1-4 bytes, normally 3 bytes.

LTE-Sim allows to simulate a heterogeneous traffic over LTE network. Therefore, it is quite similar to a real system. The outputs of this software including 


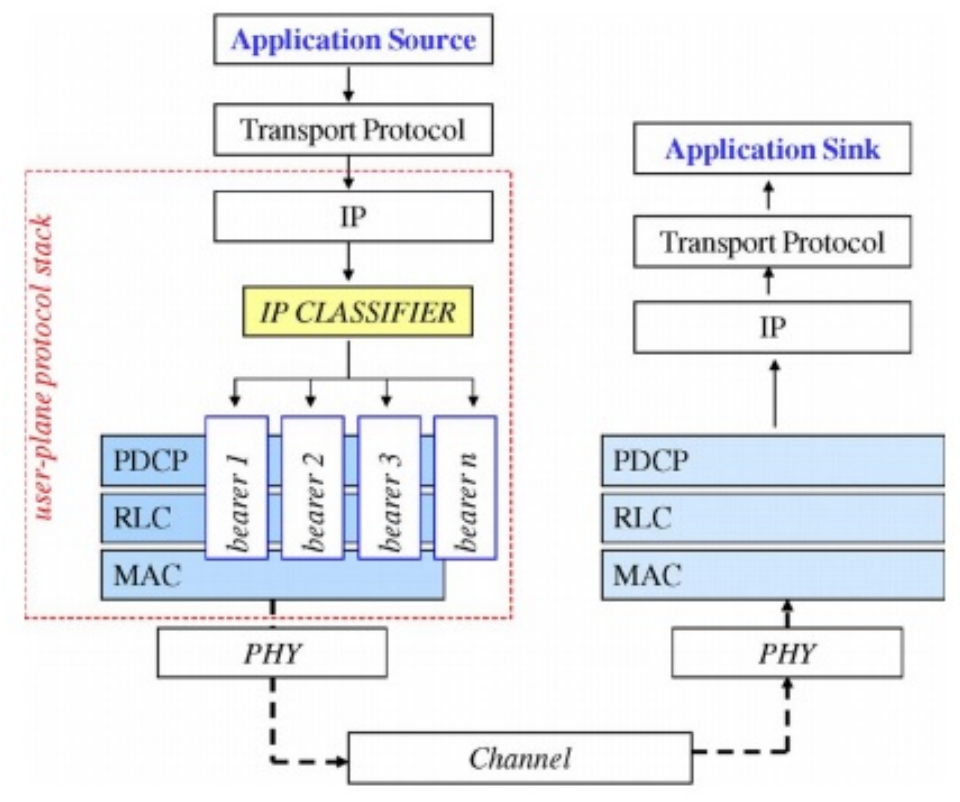

Fig. 1. The implemented protocol stack in LTE-Sim [15]

many parameters where there are delay and PLR. LTE-Sim supports multicell/multiuser with mobility in a heterogeneous network. The details of the simulation scenario is represented in Section 3.

\subsection{The extended E-model}

E-model is a computational model developed and standardized by ITU-T [5]. It is used to estimate the MOS for narrow band audio quality. The output of the model is R-factor. The values of this R-factor in range of 0-100 where 100 is the best and 0 is the worst quality. And then, it is mapped to the corresponding MOS value. The standard R-factor in the E-model is defined as follows:

$$
R=R_{0}-I_{s}-I_{d}-I_{e f}+A
$$

In which: $R_{0}$ : The basic signal-to-noise ratio which consists of noise sources such as circuit and room noise. In this model, its value is set to 94.2. $I_{s}$ : The simultaneous impairment factor, it is the sum of all impairments which may occur more or less simultaneously with the voice transmission. In this model, the default value is set to $0 . I_{d}$ : The delay impairment factor, representing all impairments due to delay of voice signals. $I_{e f}$ : The equipment impairment factor, capturing the effect of signal distortion due to low bit rates of the codec and packet losses of random distribution. A: The advantage factor, capturing the fact 
that some users can accept a reduction of quality due to the mobility of cellular networks. In this model, this factor is set to 0 .

In above factors, $I_{d}$ and $I_{e f}$ are affected by end-to-end delay and packet loss, respectively, while $R_{0}$ and $I_{s}$ do not depend on network performance. The R-factor is then translated into the MOS as follows [5]:

$$
\text { MOS }=\left\{\begin{array}{l}
1, \text { if } R<0 \\
1+0.035 \times R+7 \times 10^{-6} \times R \times(R-60) \times(100-R), \\
\text { if } 0 \leq R \leq 100 \\
4.5, \text { otherwise }
\end{array}\right.
$$

The relation between R-factor, user perception, and MOS is described in the Table 2.

Table 2. R-factor and MOS with corresponding user satisfaction

\begin{tabular}{|c|l|c|}
\hline$R$ & \multicolumn{1}{|c|}{ User satisfaction } & MOS \\
\hline $90 \leq R<100$ & Very satisfied & $4.3-5.0$ \\
\hline $80 \leq R<90$ & Satisfied & $4.0-4.3$ \\
\hline $70 \leq R<80$ & Some users dissatisfied & $3.6-4.0$ \\
\hline $60 \leq R<70$ & Many users dissatisfied & $3.1-3.6$ \\
\hline $50 \leq R<60$ & Nearly all users dissatisfied & $2.6-3.1$ \\
\hline$R<50$ & Not recommended & $<2.6$ \\
\hline
\end{tabular}

After setting the default values for the E-model, Equation (1) can be rewritten as follows:

$$
R=94.2-I_{d}-I_{e f}
$$

We see that, when voice packet transmitted over an IP network, it is affected by many network impairments such as PLR, delay, jitter, etc. In the E-model, there is no presence of network jitter. In order to improve use satisfaction, we propose to add the $I_{j}$ factor to the E-model. It's obvious, when add $I_{j}$ to the E-model, user perception of the extended E-model will be lower than E-model, and the E-model can be described as the following formula:

$$
R=94.2-I_{d}-I_{e f}-I_{j}
$$

Equation (4) shows that the R-factor depends on end-to-end delay $\left(I_{d}\right)$, total loss probability $\left(I_{e f}\right)$, and network jitter $\left(I_{j}\right)$. Hence, in order to compute the R-factor, we must to count these factors. The $I_{d}$ is a factor which is affected by end-to-end delay and is calculated as follows [16]:

$$
I_{d}=0.024 \times d+0.11 \times(d-177.3) \times H(d-177.3)
$$

In which: $H(x)$ is the Heavyside function:

$$
H(x)=\left\{\begin{array}{l}
0, \text { if } x<0 \\
1, \text { otherwise }
\end{array}\right.
$$


In equation (5), $d$ represents the total end-to-end delay (or mouth-to-ear delay) of speech packet. It is one of output results of LTE-Sim software. The $I_{e f}$ is determined according to packet loss. In order to compute this factor, we use the equation in [17] as follows:

$$
I_{e f}=\lambda_{1}+\lambda_{2} \times \ln \left(1+\lambda_{3} \times e_{l}\right)
$$

Where: The $\lambda_{1}$ represents the voice quality impairment factor caused by the encoder, $\lambda_{2}$ and $\lambda_{3}$ represent the effect of loss on voice quality for a given codec. Such that, these factors depend on the voice codec used. In this study, we use LTE-Sim [15] to simulate. This simulation tool supports only G.729 codec, thus, for this codec, the factors above has values as follows: $\lambda_{1}=11, \lambda_{1}=40, \lambda_{3}=10$. While $e_{l}$ is the total loss probability (consisting of network and buffer layout) which has the value in range of $0 . .1$. This factor is also obtained from the output results of LTE-Sim software.

The $I_{j}$ represents the impacts of network jitter to voice quality. It also depends on the voice codec. In this paper, we use the method proposed in [10] as follows:

$$
I_{j}=C_{1} \times H^{2}+C_{2} \times H+C_{3}+C 4 \times \exp \left(\frac{-T}{K}\right)
$$

In which: $C_{1}, C_{2}, C_{3}, C_{4}$ are coefficients, $\mathrm{K}$ is time instant. These factors depend on the voice codec, for the G.729 codec, these factors have the values as follows: $C_{1}=-15.5, C_{2}=33.5, C_{3}=4.4, C_{4}=13.6, K=30$. The factor of $\mathrm{T}$ is the fixed buffer size of the voice codec. For the G.729 codec, the packet size is $20 \mathrm{~ms}$, thus, normally $T=\propto \times 20$ where $\propto=2,3,4,5,6$, etc. The $H$ is a factor of Pareto distribution and in range of 0.55 to 0.9. According to [10], the MOS slightly drops when $H$ increases and it does not affect significantly on MOS score, thus, in this study, we select $\mathrm{H}=0.6$ for the simulation.

The final expression of the R-factor when utilizing the G.729 codec is described in Equation (9).

$$
\begin{aligned}
& R=64.28-[0.024 \times d+0.11 \times(d-177.3) \times H(d-177.3)]- \\
& 40 \times \ln \left(1+10 \times e_{l}\right)-13.6 \times \exp \left(\frac{-4}{3}\right)
\end{aligned}
$$

The R-factor is then mapped to the MOS via equations (2). MOS reflects the perception of user. It is obvious, the higher MOS, the higher user satisfaction and inverse. How to determine exactly the perception of voice user is a big challenge of mobile operators.

\section{The proposed model}

Voice over LTE network (VoLTE) is a real-time service, and is fully deployed over an IP network, thus, in order to ensure VoLTE quality is very complex. There are very little methods that allow to monitor and to predict VoLTE quality and most of them are subjective ones which have to refer to the original signal, thus, they are not suitable for real-time services such as VoIP, Video, Gaming, etc. In this paper, we propose a new non-intrusive voice quality assessment method 
which based on LTE-Sim software [15] and the extension of the E-model [5]. LTE-Sim is a software which allow to simulate VoIP flow that is quite similar to real flow. Therefore, we see that, the simulation results are quite exact. The E-model is a computational model which allows to predict voice quality when it is transmitted from source to destination. However, in this model, there is not presence of network jitter. For VoIP flow, network jitter affects significantly on voice quality, thus, we propose to add the effects of network jitter to E-model via $I_{j}$ factor. When the $I_{j}$ is added to the E-model, the value of $R$ factor will be lower, it leads to the lower user perception when compared to the standard E-model. The proposed model is represented on Figure 2.

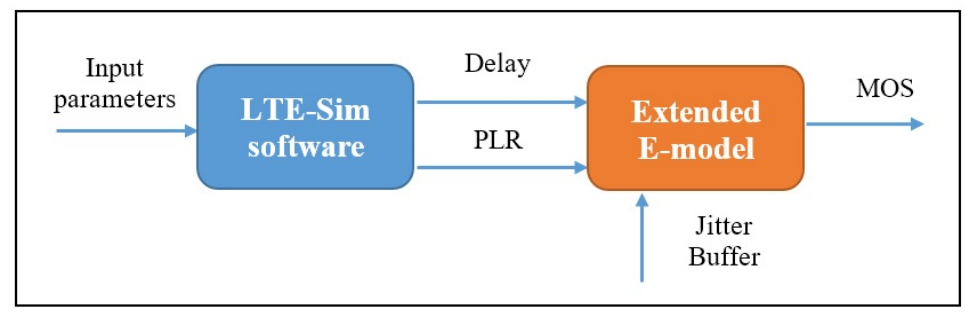

Fig. 2. The proposed implement model

The principle of the proposed model as follows: Input parameters of the simulation scenarios are firstly fed to the LTE-Sim software. After finishing the simulation, we receive simulation results. In these results, we select Delay, PLR according to the number of user. These factors combining jitter buffer (JB) are the input parameters of the extended E-model. The output of E-model is $R$ factor, and then it is mapped to MOS score. This MOS score performs the user satisfaction. The steps of the proposed model can be described as follows:

- Step 1: Setting input parameters of the simulation scenario.

- Step 2: Simulating the scenario in the LTE-Sim software

- Step 3: Collecting the delay, PLR results from Step 2, and selecting values of JB.

- Step 4: Feeding the input parameters taken from Step 3 to the Extended E-model which is programmed in Matlab software [18] as follows:

- Calculating $R$ factor of the extended E-model according to Equation (9).

- $R$ is then mapped to MOS score based on Formula (2).

After Step 4, we will receive the user satisfactions according to the number of user.

LTE is a packet-switched network, thus, in order to simulate it as a real system, we select input data flows including a VoIP, a Video and a non real-time flow. In addition, the mobility is also included in our scenario, specifically, we select the speed of user is 30 and $120 \mathrm{~km} / \mathrm{h}$. We select the M-LWDF scheduler 
which is very suitable for real-time services. Besides predicting the user perception, we assess the effects of the Delay, PLR on user satisfaction according to the number of user.

\section{Simulation environment and Performance evaluation}

\subsection{Simulation environment}

Traffic model: In our scenario, the eNB is located at the center of the macrocell using an ommi-directional antenna in a $10 \mathrm{MHz}$ bandwidth. Each UE uses a VoIP flow, a Video flow, and a INF-BUF flow at the same time. For the VoIP flow, a G.729 voice stream with a bit-rate of $8 \mathrm{kbps}$ was considered. The voice flow is a bursty application that is modelled with an ON/OFF Markov chain [19]. For the video flow, a trace-based application that generates packets based on realistic video trace files with a bit-rate of $242 \mathrm{kbps}$ was used in [20] and it is also available in [15]. In order to obtain a realistic simulation of an H.264 SVC video streaming, we used an encoded video sequence "foreman.yuv", which is publicly available. The LTE propagation loss model is formed by four different models including: Path loss, Multipath, Penetration and Shadowing [21].

- Path loss: $P L=128.1+37.6 \times \log (d)$, with $\mathrm{d}$ is the distance between the $\mathrm{UE}$ and the eNB in $\mathrm{km}$.

- Multipath: Jakes model

- Penetration loss: $10 \mathrm{~dB}$

- Shadowing: Log-normal distribution with mean $0 \mathrm{~dB}$ and standard deviation of $8 \mathrm{~dB}$.

Simulation parameters: In this paper, we use M-LWDF scheduler for simulating. This scheduler is very suitable for voice traffic flow. The simulation process is performed in a single cell with interference with the number of users in the interval $[10,50]$ which move randomly at a speed of 30 and $120 \mathrm{~km} / \mathrm{h}$. The other basic parameters used in the simulation are represented in the Table 3.

\subsection{Performance evaluation}

In order to simulate the traffic model in LTE-Sim, we use Modified Largest Weighted Delay First (M-LWDF) [22] scheduler with mobility in LTE heterogeneous network. The M-LWDF scheduler is very suitable for real-time services such as VoIP, Video, Gaming, etc. The analyses of the simulation results are represented in the following subsections.

Effects of Jitter buffer on voice quality: In order to evaluate the effects of JB on voice quality, we set values of this factor of 40,60,80, 100, and 120 . Figure 3 shows the relationship of the number of user (NU) vs user perception 
Table 3. Simulation parameters

\begin{tabular}{|l|l|}
\hline Simulation Parameters & \multicolumn{1}{|c|}{ Values } \\
\hline Simulation duration & $100 \mathrm{~s}$ \\
\hline Frame structure & FDD \\
\hline Cell radius & $1 \mathrm{~km}$ \\
\hline Bandwidth & $10 \mathrm{MHz}$ \\
\hline Video bit-rate & $242 \mathrm{kbps}$ \\
\hline VoIP bit-rate & $8 \mathrm{kbps}$ \\
\hline User speed & $30,120 \mathrm{~km} / \mathrm{h}$ \\
\hline Number of user & $10,20,30,40,50$ UEs \\
\hline Maximum delay & $0.1 \mathrm{~s}$ \\
\hline Packet Scheduler & M-LWDF \\
\hline Traffic model & VoIP, Video, and INF-BUF \\
\hline
\end{tabular}

(MOS score) when the speed is $30 \mathrm{~km} / \mathrm{h}$. It's clear that, in this case, the Emodel has the highest MOS score while the proposed model has the lower MOS. This is obvious because our proposed model considers effect of network jitter on the E-model. All cases have the slightly degraded MOS when NU increases. This figure also shows that the higher JB, the higher MOS. However, in fact, JB should not be too high. As shown on this figure, when JB is more than 80 , the MOS increase not significantly. This is quite clear when JB corresponds to 100 and 120. It can be said that, when JB is more than 100, its effect on user perception is not significant.

Figure 4 shows the effects of JB when the speed is $120 \mathrm{~km} / \mathrm{h}$. It's clear that, when the speed is high, the user satisfaction is decreased clearly. All cases have the MOS score heavily decreasing when NU increasing. It's obvious, but the reduction is quite different from the case of the speed of $30 \mathrm{~km} / \mathrm{h}$. It's similar to the previous case, the higher JB, the higher user perception. The JB is directly proportional to user satisfaction while the speed is inverse.

Effects of Delay on voice quality: Figure 5 shows the relationship between delay vs user perception according to NU. It's clear that, the delay increases when NU increase. This leads to lower MOS score. The E-model has the highest MOS while the Extended E-model has the lower one. It can be said that, the higher NU and the higher delay, the lower MOS score (or lower user perception). This principle is suitable for both the E-model and the Extended E-model (for all cases of JB). The detailed results of this case are represented in Table 4.

It's similar to the case of delay for the user speed of $30 \mathrm{~km} / \mathrm{h}$, the effects of delay on user satisfactions when the user speed is $120 \mathrm{~km} / \mathrm{h}$ is shown on Figure 6 . The principle is similar to the user speed of $30 \mathrm{~km} / \mathrm{h}$, however, the MOS score is lower and heavily decreases when the NU or delay increases. This is right in both E-model and Extended E-model. The detailed results are described in Table 4 . 


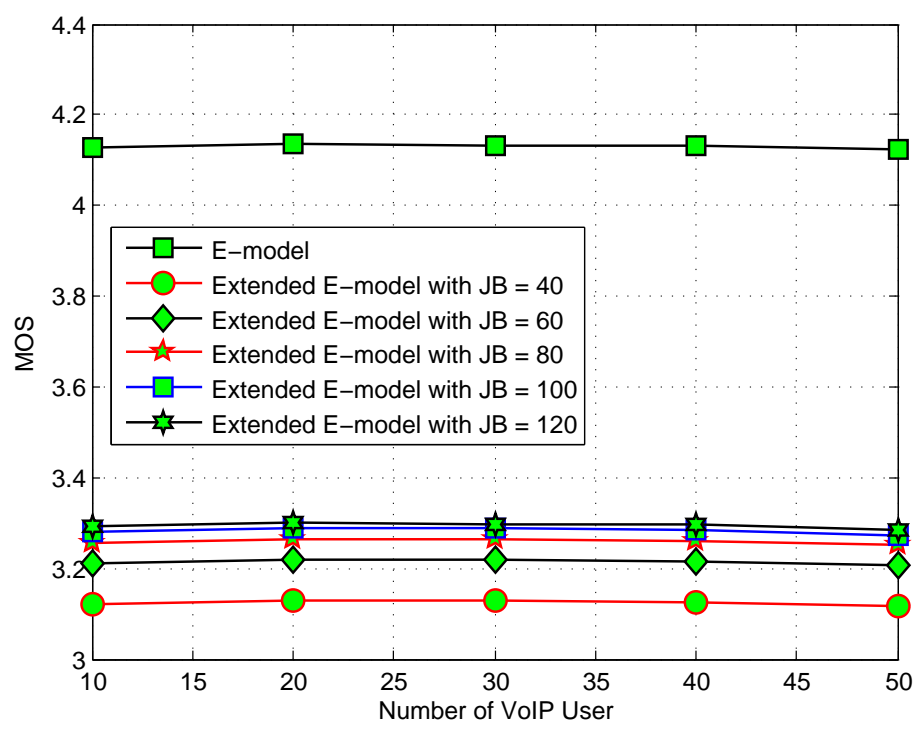

Fig. 3. MOS vs number of VoIP user at the speed of $30 \mathrm{~km} / \mathrm{h}$

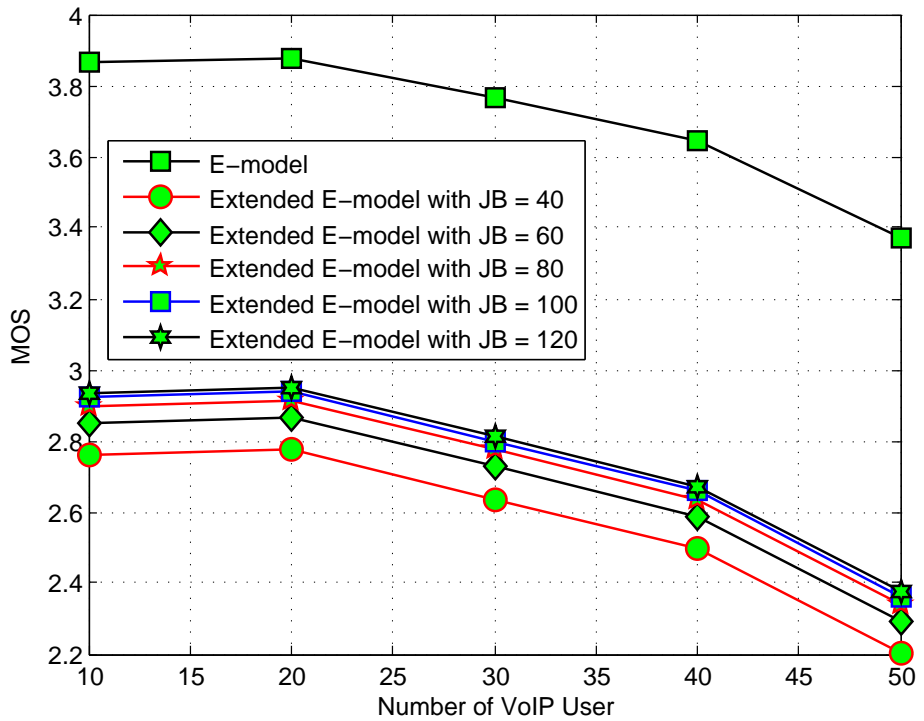

Fig. 4. MOS vs number of VoIP user at the speed of $120 \mathrm{~km} / \mathrm{h}$ 


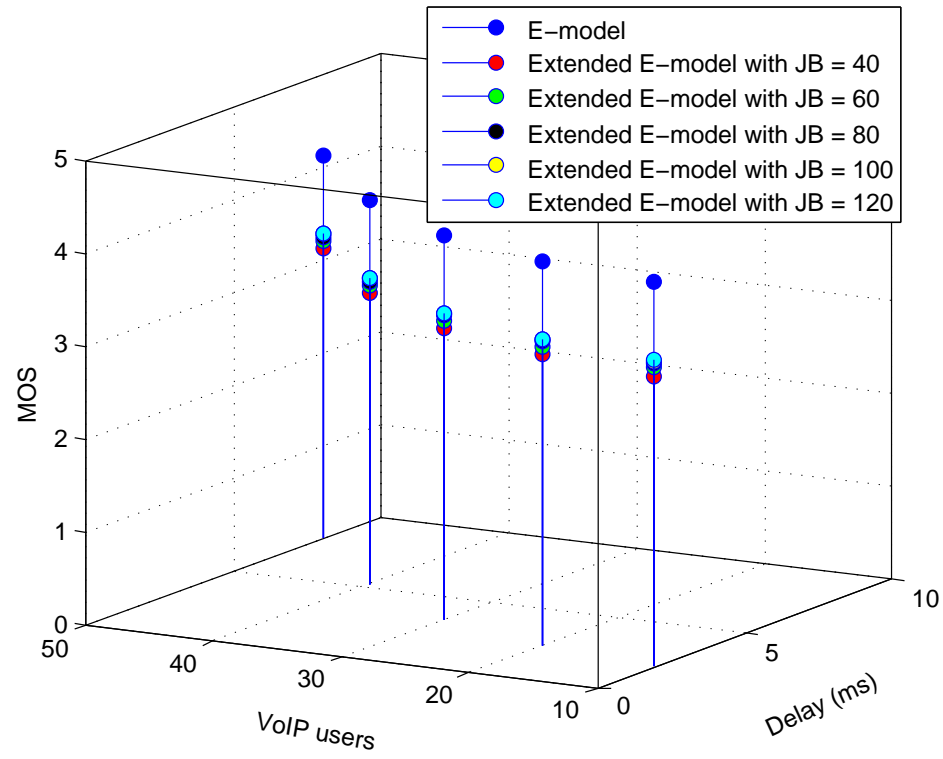

Fig. 5. Effects of Delay on voice quality at the speed of $30 \mathrm{~km} / \mathrm{h}$

Effects of PLR on voice quality: Figure 7 illustrates the effects of delay on user perception according to NU. It's clear that, MOS increases when the NU or PLR increases. This is suitable for both E-model and extended E-model. Normally, PLR increases when NU increases, but there is special case for the NU equals 10 . This may be due to the system is usually not stable in fact. For both E-model and Extended E-model, the higher NU and the higher PLR, the lower MOS score. The E-model has the higher MOS when compared to the Extended E-model. For the Extended E-model, rule for the higher JB, the higher MOS is still met. For the detailed results, refer to Table 4.

For the case of the user speed equals $120 \mathrm{~km} / \mathrm{h}$, the rules for the case of user speed is $30 \mathrm{~km} / \mathrm{h}$ is still met. The difference here is that in this case, the user perception decreases in comparison with the user speed of $30 \mathrm{~km} / \mathrm{h}$ and it heavily decreases when the NU or PLR increases. This is fully suitable. The detailed results of this case are represented in Table 4 . 


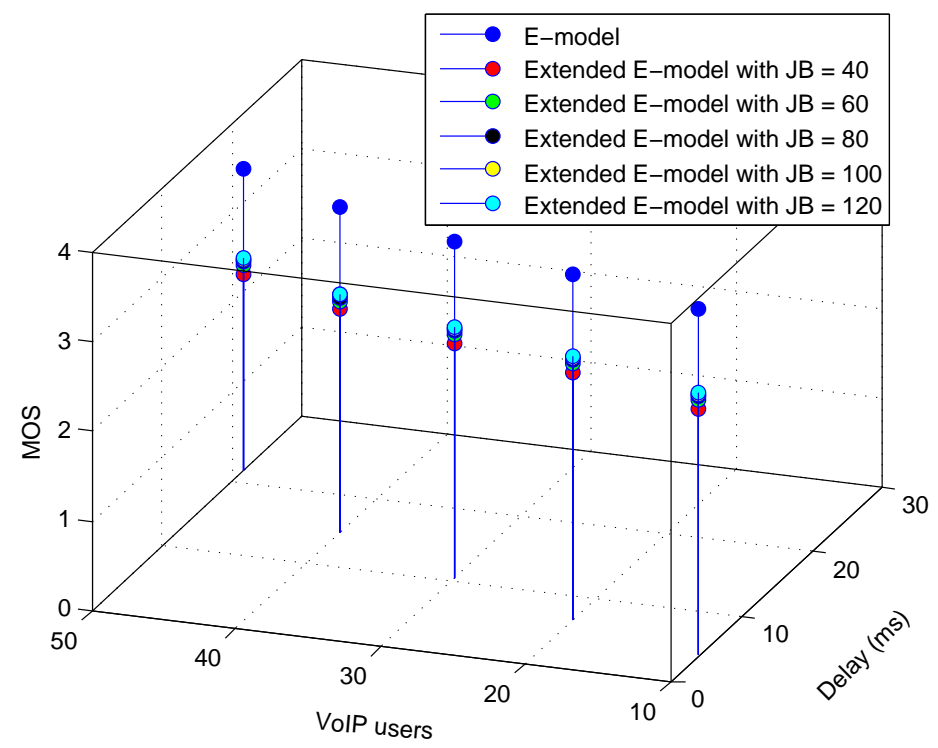

Fig. 6. Effects of Delay on voice quality at the speed of $120 \mathrm{~km} / \mathrm{h}$

Table 4. The detailed results of Figures of $5-8$

\begin{tabular}{|c|c|c|c|c|c|c|c|c|}
\hline \multirow{3}{*}{$\mathbf{N U}$} & \multirow{3}{*}{$\begin{array}{c}\text { Delay } \\
(\mathbf{m s})\end{array}$} & \multirow{3}{*}{$\begin{array}{c}\text { PLR } \\
(\%)\end{array}$} & \multicolumn{6}{|c|}{ MOS } \\
\hline & & & \multirow{2}{*}{ E-model } & \multicolumn{5}{|c|}{ Extended E-model } \\
\hline & & & & \multicolumn{5}{|c|}{\begin{tabular}{|l|l|l|l|l|}
$\mathbf{J B}=40$ & $\mathbf{J B}=\mathbf{6 0}$ & $\mathbf{J B}=\mathbf{8 0}$ & $\mathbf{J B}=\mathbf{1 0 0}$ & $\mathbf{J B}=\mathbf{1 2 0}$ \\
\end{tabular}} \\
\hline \multicolumn{9}{|c|}{ User speed: $30 \mathrm{~km} / \mathrm{h}$} \\
\hline 10 & 1.94 & 0.064 & 1287 & 3.1204 & 3.21 & 3.2556 & 3.2789 & 3.2909 \\
\hline 20 & 2.47 & 0.014 & 351 & 3.13 & 3.2195 & 3.2651 & 3.2884 & 3.3003 \\
\hline 30 & 45 & 7 & & 3.1282 & 3.2177 & 3.2633 & .2886 & 3.2985 \\
\hline 40 & 5.26 & 0.018 & 322 & 3.1257 & 3.2153 & 2.2609 & 3.2842 & 3.2961 \\
\hline 50 & 33 & 3 & 252 & 3.1151 & 3.2048 & 3.2504 & 3.2738 & 3.2857 \\
\hline \multicolumn{9}{|c|}{ User speed: $120 \mathrm{~km} / \mathrm{h}$} \\
\hline 10 & 4.04 & 1.943 & 3.8643 & 2.7591 & 2.851 & 2.8981 & 2.9223 & 2.9347 \\
\hline 20 & 6.64 & 1.833 & & 2.7753 & 2.8672 & 2.9143 & 2.9385 & 2.9508 \\
\hline 30 & 10.36 & 2.618 & 3.7632 & 2.6352 & 2.7271 & 2.7743 & 2.7986 & 2.811 \\
\hline 40 & 14.74 & 3.448 & 3.6434 & 2.496 & 2.5874 & 2.6345 & 2.6587 & 2.6711 \\
\hline 50 & 21.54 & 5.432 & 3.371 & 2.2044 & 2.293 & 2.3389 & 2.3626 & 2.3748 \\
\hline
\end{tabular}




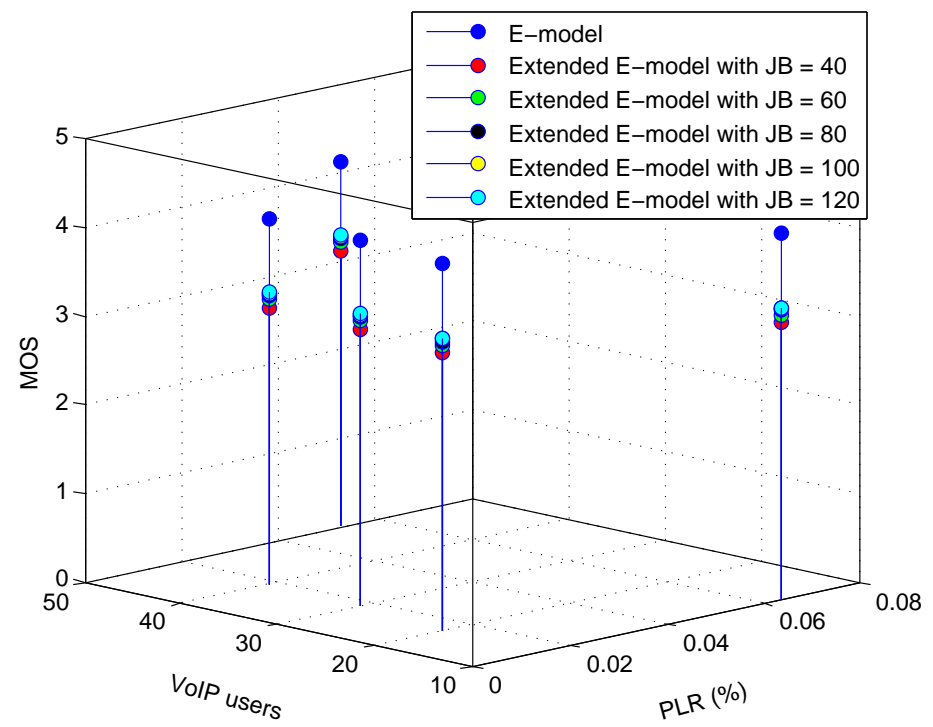

Fig. 7. Effects of PLR on voice quality at the speed of $30 \mathrm{~km} / \mathrm{h}$

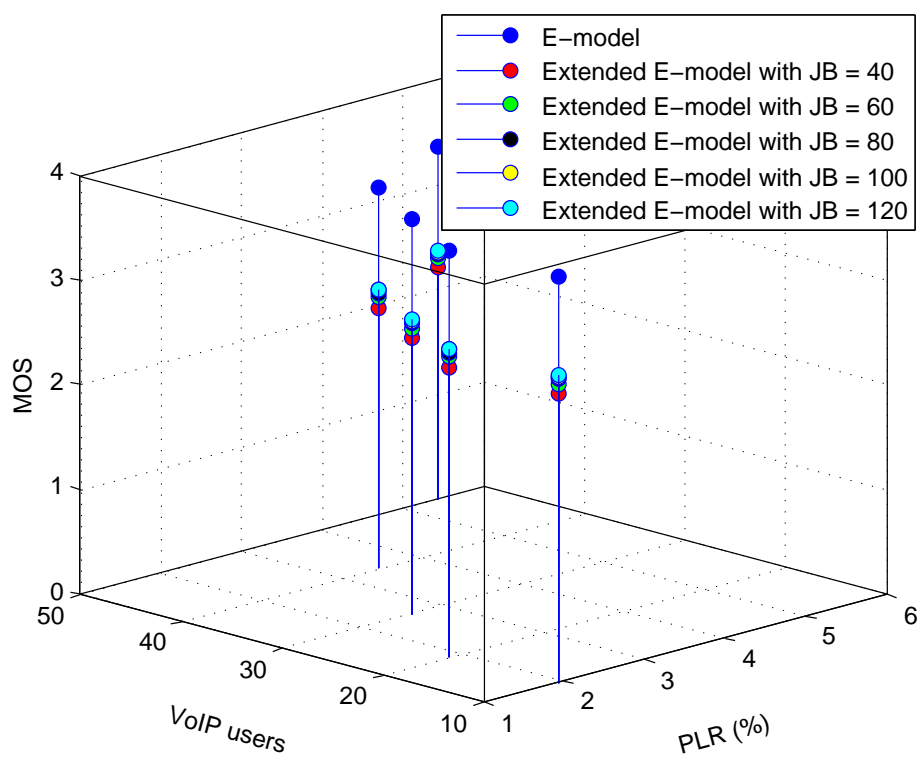

Fig. 8. Effects of PLR on voice quality at the speed of $120 \mathrm{~km} / \mathrm{h}$ 


\section{Conclusion}

In this study, we propose a new non-intrusive assessment method of voice quality over LTE network. Our method is the combination of the LTE-Sim framework and the extension of the E-model. The simulation results show that, our proposed model has the lower user perception in comparison with the E-model one. This is because in our model, we take effects of network jitter into account. This also means the effect of JB on user perception is very significant. The simulation results also show that user perception decreases when the user speed increases. For the case of user speed of $30 \mathrm{~km} / \mathrm{h}$, the user satisfaction slightly reduces when $\mathrm{NU}$ raises while it heavily decreases for the user speed of $120 \mathrm{~km} / \mathrm{h}$. For both cases of the user speed, the user perception increases when the JB increases. However, when JB is more than 100, the user satisfaction increases not significantly. For the delay and PLR, both increase when NU increases and meet the principle: the higher delay and PLR, the lower user satisfaction. It can be concluded that, the proposed model is very suitable for predicting VoLTE quality be cause it does not refer to the original signal. The user perception can be not as exact as the intrusive methods such as PESQ but it is quite simple, calculate very quickly. It is also very suitable for purpose of transmission planning and for voice assessment in laboratory for academic community and researchers. The proposed model can be used to predict VoLTE quality for many different scenarios which can be configured in the LTE-Sim. In the near future, we will evaluate our proposed model for more NU, other voice codecs, and complement more parameters into the E-model.

\section{References}

1. 3rd Generation Partnership Project (3GPP). Avaiable:http://www .3gpp.org

2. Hyun, J., Li, J., Im, C., Yoo, J.H., Hong, J.W.K.: A volte traffic classification method in lte network. In: Network Operations and Management Symposium (APNOMS), 2014 16th Asia-Pacific, IEEE (2014) 1-6

3. ITU-T: Itu-t recommendation p.800: Methods for subjective determination of transmission quality. Technical report, International Telecommunication Union (August 1996)

4. ITU-T: Itu-t recommendation p.862: Perceptual evaluation of speech quality (pesq): An objective method for end-to-end speech quality assessment of narrowband telephone networks and speech codecs. Technical report, International Telecommunication Union (February 2001)

5. ITU-T: Itu-t recommendation g.107: The e-model, a computational model for use in transmission planning. Technical report, International Telecommunication Union (December 1998)

6. Radhakrishnan, K., Larijani, H., Buggy, T.: A non-intrusive method to assess voice quality over internet. In: Performance Evaluation of Computer and Telecommunication Systems (SPECTS), 2010 International Symposium on, IEEE (2010) $380-386$

7. Larijani, H., Radhakrishnan, K.: Voice quality in voip networks based on random neural networks. In: Networks (ICN), 2010 Ninth International Conference on, IEEE (2010) 89-92 
8. Ghalut, T., Larijani, H.: Non-intrusive method for video quality prediction over lte using random neural networks (rnn). In: Communication Systems, Networks \& Digital Signal Processing (CSNDSP), 2014 9th International Symposium on, IEEE (2014) 519-524

9. Duy-Huy Nguyen, H.N.: A dynamic rate adaptation algorithm using wb e-model for voice traffic over lte network. In: Wireless days (WD), 2016 9th IFIP, IEEE (2016)

10. Ding, L., Goubran, R.A.: Speech quality prediction in voip using the extended emodel. In: Global Telecommunications Conference, 2003. GLOBECOM'03. IEEE. Volume 7., IEEE (2003) 3974-3978

11. Meddahi, A., Afifl, H., Zeghlache, D.: " packet-e-model": e-model for wireless voip quality evaluation. In: Personal, Indoor and Mobile Radio Communications, 2003. PIMRC 2003. 14th IEEE Proceedings on. Volume 3., IEEE (2003) 2421-2425

12. Carvalho, L., Mota, E., Aguiar, R., Lima, A.F., Barreto, A., et al.: An e-model implementation for speech quality evaluation in voip systems. In: null, IEEE (2005) 933-938

13. Takahashi, A., Kurashima, A., Yoshino, H.: Objective assessment methodology for estimating conversational quality in voip. Audio, Speech, and Language Processing, IEEE Transactions on 14(6) (2006) 1984-1993

14. Assem, H., Malone, D., Dunne, J., O'Sullivan, P.: Monitoring voip call quality using improved simplified e-model. In: Computing, networking and communications (ICNC), 2013 international conference on, IEEE (2013) 927-931

15. Piro, G., Grieco, L.A., Boggia, G., Capozzi, F., Camarda, P.: Simulating lte cellular systems: an open-source framework. Vehicular Technology, IEEE Transactions on 60(2) (2011) 498-513

16. Olariu, C., Foghlu, M.O., Perry, P., Murphy, L.: Voip quality monitoring in lte femtocells. In: Integrated Network Management (IM), 2011 IFIP/IEEE International Symposium on, IEEE (2011) 501-508

17. Mushtaq, M.S., Augustin, B., Mellouk, A.: Qoe-based lte downlink scheduler for voip. In: Wireless Communications and Networking Conference (WCNC), 2014 IEEE, IEEE (2014) 2190-2195

18. The Mathworks, Inc. Natick, Massachusetts: MATLAB version 8.5.0.197613 (R2015a). (2015)

19. Chuah, C.N., Katz, R.H.: Characterizing packet audio streams from internet multimedia applications. In: Communications, 2002. ICC 2002. IEEE International Conference on. Volume 2., IEEE (2002) 1199-1203

20. Reisslein, M., Karam, L., Seeling, P.: H. 264/AVC and SVC Video Trace Library: A Quick Reference Guide http://trace. eas. asu. edu. (2009)

21. 3GPP: Requirements for Evolved UTRA (E-UTRA) and Evolved UTRAN (EUTRAN). TR 25.913, 3rd Generation Partnership Project (3GPP) (12 2009)

22. Ameigeiras, P., Wigard, J., Mogensen, P.: Performance of the m-lwdf scheduling algorithm for streaming services in hsdpa. In: Vehicular technology conference, 2004. VTC2004-Fall. 2004 IEEE 60th. Volume 2., IEEE (2004) 999-1003 